eISSN 2444-7986

DOI: https://doi.org/10.14201/orl201674.14699

Caso clínico

\title{
IMPLANTE COCLEAR EN ENFERMEDAD DE MÉNIÈRE BILATERAL. DESCRIPCIÓN DE UN CASO
}

\section{Cochlear implantation in bilateral Ménière's disease. A case report}

\author{
Ainhoa MORENO-BRAVO'; Hortensia SÁNCHEZ-GÓMEZ²; Gabriel Alejandro AGUILERA- \\ AGUILERA'; ${ }^{2}$ Myriam GONZÁLEZ-SÁNCHEZ ${ }^{3}$; Santiago SANTA CRUZ-RUIZ²; Ángel \\ BATUECAS-CALETRIOO ${ }^{2}$
}

${ }^{1}$ Complejo Hospitalario de Mérida. Servicio de Otorrinolaringología y Patología Cérvico-facial. Mérida. España. ${ }^{2}$ Complejo Asistencial Universitario de Salamanca. Servicio de Otorrinolaringología y Patología Cérvico-facial. Salamanca. España. ${ }^{3}$ Hospital Virgen de la Concha. Servicio de Otorrinolaringología y Patología Cérvico-facial. Zamora. España.

Correspondencia: amb-huertas@hotmail.com

Fecha de recepción: 7 de junio de 2016

Fecha de aceptación: 13 de julio de 2016

Fecha de publicación: 15 de julio de 2016

Fecha de publicación del fascículo: 1 de marzo de 2017

Conflicto de intereses: Los autores declaran no tener conflictos de intereses

Imágenes: Los autores declaran haber obtenido las imágenes con el permiso de los pacientes

Política de derechos y autoarchivo: se permite el autoarchivo de la versión post-print (SHERPA/RoMEO)

Licencia CC BY-NC-ND. Licencia Creative Commons Atribución-NoComercial-SinDerivar 4.0 Internacional

(C) Universidad de Salamanca. Su comercialización está sujeta al permiso del editor

RESUMEN

Introducción: La enfermedad de Ménière se caracteriza por síntomas cocleares y vestibulares. Puede ser unilateral o bilateral. Cuando la pérdida de audición asociada es severa-profunda de forma bilateral o unilateral pero con problemas de audición en el otro oído, dicha audición puede beneficiarse de un implante coclear. Si además, presenta crisis de vértigo y Tumarkin refractarias al tratamiento médico, se puede plantear la realización simultánea de una laberintectomía quirúrgica y colocación de un implante coclear. Descripción: Se describe el caso de un paciente con enfermedad de Ménière bilateral con hipoacusia neurosensorial profunda de oído derecho con fluctuaciones auditivas izquierdas que asocia crisis vertiginosas y de Tumarkin sin respuesta a corticoides orales e intratimpánicos ni a gentamicina intratimpánica. Se le realizó una laberintectomía quirúrgica con implante coclear en el mismo acto quirúrgico anticipándonos al probable deterioro auditivo contralateral. Posteriormente presenta buen rendimiento del implante y desaparición de las crisis del oído intervenido. Discusión: El objetivo del tratamiento es controlar las crisis preservando la función, pero en pacientes en los que las medidas conservadoras fallan, se han de considerar otras más destructivas como es la laberintectomía quirúrgica, que consigue altas tasas de control de las crisis, y el implante coclear, indicado para restaurar la audición en el oído afectado por enfermedad de Ménière. Si ambas se realizan en un mismo tiempo quirúrgico, se consigue reducir riesgos asociados con otros procedimientos quirúrgicos y anestésicos y evitar las modificaciones histológicas tras una laberintectomía que dificultarían la posterior colocación del implante coclear.

PALABRAS CLAVE enfermedad de Ménière; hipoacusia bilateral; crisis de Tumarkin; implante coclear 
Introduction and objective: Ménière's disease (MD) is an episodic vestibular syndrome associated with sensorineural hearing loss and tinnitus. It may be unilateral or bilateral. Cochlear implantation $(\mathrm{Cl})$ could be considered in MD patients if a profound hearing loss is showed in both ears. As alternative, it should be valued if profound hearing loss affects only one ear but the contralateral is affected too. When a MD patient suffers from vertigo spells or otolitic crisis of Tumarkin appart from the profound hearing loss, a simultaneous surgical labyrinthectomy and $\mathrm{Cl}$ procedure could be considered. Description: Bilateral MD patient with right profound hearing loss and left fluctuating moderate hearing loss. No response was observed after oral and intratympanic steroids and intratympatic gentamicin in the treatment of right ear. A cochlear implantation and classic labyrinthectomy were performed at the same time in the right ear. No new vertigo spells or otolitic crisis of Tumarkin appeared after three months of follow up. Discussion: The objective in the treatment of MD is to get the control of vertigo spells and a hearing preservation. When conservative treatment is not useful, the gold standard is the labyrinthectomy (gentamicin or classic labyrinthectomy). The surgical labyrinthectomy could be accompanied by cochlear implantation to restore hearing in this ear. To perform both surgical labyrinthectomy and cochlear implantation simultaneously should be considered in order to reduce anesthesic ricks and cochlear histological changes after labyrinthectomy.

\section{INTRODUCCIÓN}

La comunicación efectiva es necesaria. Para un joven es uno de los aspectos de la vida más importantes, sin embargo, debido a la enfermedad de Ménière (EM), dicha comunicación se ve alterada influenciando en su vida diaria. $\mathrm{Si}$ a esto sumamos los síntomas derivados de las crisis de vértigo propias de la enfermedad, puede llegar a incapacitar y llevar a estados de depresión y ansiedad [1].

La EM tiene una incidencia de 4,3 a 15,3 casos por 100.000 habitantes al año [2-5] y su etiología es idiopática pero existe una clara asociación con el hídrops endolinfático, un aumento de la presión endolinfática en el laberinto membranoso del oído afecto [6].

La EM unilateral se caracteriza por síntomas cocleares y vestibulares como fluctuaciones de audición tipo neurosensorial en frecuencias graves, crisis de vértigo, acúfenos y plenitud ótica [7]. Menos frecuente es la variante bilateral cuyos síntomas incluyen los clásicos del unilateral y asocia desequilibrio, oscilopsia y pérdida audición bilateral pudiendo tener mayor impacto en la calidad de vida del paciente. La mayoría de los casos comienzan por la aparición de síntomas en un oído y secuencialmente evolucionan hacia la enfermedad bilateral. La frecuencia de conversión de unilateral a bilateral no está clara, varios estudios lo sitúan entre el $5 \%$ y $50 \%$ dependiendo de los criterios utilizados audiológicos y de la duración del seguimiento $[8,9]$. Por lo general, el número de pacientes con EM que progresan a forma bilateral con hipoacusia neurosensorial (HNS) severa o profunda bilateral es relativamente pequeña, aproximadamente un $6 \%$ [8]. El periodo de conversión es otro punto de controversia, algunos autores sostienen que los síntomas contralaterales aparecen entre 2 y 5 años desde la aparición inicial [10].No es frecuente predecir qué pacientes progresarán a una enfermedad bilateral a pesar de los resultados de múltiples trabajos que estudian la capacidad predictiva de la electrococleografía [11], los potenciales evocados miogénicos vestibulares [12] y la resonancia de alta resolución con gadodiamida intratimpánica [13].

El curso clínico de la EM varía entre pacientes. Unos con marcados síntomas vestibulares y mínima pérdida auditiva, mientras que otros experimentan principalmente pérdida de audición con problemas vestibulares leves. Sin embargo, otros pueden estar igualmente afectados en cuanto a la audición y los síntomas vestibulares. La pérdida de audición es neurosensorial y progresiva en el inicio de la enfermedad, a menudo fluctuante, y afecta principalmente a las frecuencias graves pero en las personas con pérdida en las frecuencias altas, tiende a no ser fluctuante [14]. Progresa a lo largo del proceso de la enfermedad y, por lo general, se estabiliza con una pérdida moderada a severa [15]. Sin embargo, en una gran parte de esta población acabará progresando a un grado profundo [14]. Debido a la pérdida de ventajas de la audición binaural, los pacientes experimentan dificultad para la audición, el entendimiento y la identificación sonora. Así, cuando esto ocurre de forma bilateral o unilateral pero con problemas de audición contralateral, pueden beneficiarse de un implante coclear (IC).

EI IC es una opción reconocida y viable como terapia de rehabilitación eficaz en pacientes con sordera severa o profunda secundaria a 
EM. La estimulación eléctrica con IC restaura la percepción auditiva en el oído sordo mejorando la localización de sonidos y la percepción de las palabras en ambiente ruidoso [16]. Otra ventaja es la supresión del acúfeno [17], aunque este efecto podría estar limitado a la duración de la estimulación.

La mayoría de los pacientes con EM son manejados con medidas conservadoras: dieta, rehabilitación vestibular y medicación. Para muchos será suficiente para el control de sus síntomas manteniendo una satisfactoria calidad de vida, pero desafortunadamente un grupo de pacientes continuará con episodios frecuentes e incapacitantes para los que existe una gama de opciones a considerar como son la terapia intratimpánica con corticoides o gentamicina, la descompresión del saco endolinfático, la sección del nervio vestibular o la laberintectomía quirúrgica.

Aunque el tratamiento óptimo continúa siendo motivo de controversia, la mayoría valora el incremento de la eficacia frente al riesgo de pérdida de funcionalidad, así pues, es razonable adoptar una estrategia de tratamiento por etapas intentando suprimir los síntomas del vértigo preservando la audición, por eso los pacientes sin adecuado control del vértigo y con HNS presentan un problema terapéutico [18]. Para estos pacientes refractarios a medidas conservadoras, particularmente si han perdido audición en el oído afecto, la laberintectomía ofrece una excelente opción terapéutica. La laberintectomía quirúrgica ( $L Q x)$ transmastoidea puede ser preferible a la transcanal que provoca mayor trauma coclear [19].

Algunos pacientes con EM avanzada experimentan vértigo incapacitante y/o crisis de Tumarkin refractarias al tratamiento médico o quirúrgico no ablativo asociándose HNS de severa a profunda en oído afecto [20, 21]. La LQx ofrece altas tasas de control del vértigo en estos pacientes,sin embargo los pacientes todavía sufren las consecuencias de la hipoacusia [22, 23]. Así, en un esfuerzo por resolver los episodios de vértigo y rehabilitar los efectos de la hipoacusia, se plantea la realización de LQx simultánea con IC [24].

\section{DESCRIPCIÓN}

Paciente varón de 36 años de edad con antecedentes personales de infección por $\mathrm{VIH}$, enfermedad de Gilbert y sífilis; diagnosticado de EM de oído derecho desde hace 3 años. Con buen control de las crisis de vértigo ocasionales, hasta el último año, en el que éstas comenzaron a ser más intensas y frecuentes. Por ello se instauró tratamiento con corticoides orales primero e intratimpánicos después sin obtener respuesta. No sólo mantenía sus crisis vertiginosas, sino que a éstas se les añadían otras de Tumarkin, y la hipoacusia pasó de ser leve a profunda en ese oído derecho en los últimos meses con una logoaudiometría de umbral de inteligibilidad en 85 dB y máxima discriminación del $90 \%$ a $100 \mathrm{~dB}$ a un $10 \%$ de inteligibilidad a $100 \mathrm{~dB}$. La otoscopia era normal y las pruebas de imagen, TAC peñascos y RMN, sin hallazgos patológicos. Los estudios analíticos negativos.

Por todo esto se decidió administrar gentamicina intratimpánica, hasta 5 dosis, pasando de tener unas ganancias en el vHIT de 0,81 a 0,79 tras la primera dosis, 0,63 tras la tercera, 0,64 después de la cuarta y 0,60 tras la quinta (Figura 1). Posteriormente el paciente siguió presentando crisis de Tumarkin (aproximadamente 10 crisis cada 3 semanas). Algunas de estas crisis le ocasionaron bruscas caídas al suelo con traumatismos craneoencefálicos por los que en alguna ocasión necesitó ingreso hospitalario.

A su vez, el paciente había comenzado a notar hipoacusia y acúfeno en el oído izquierdo, que se puso de manifiesto cuando ya el derecho presentaba una hipoacusia neurosensorial moderada.

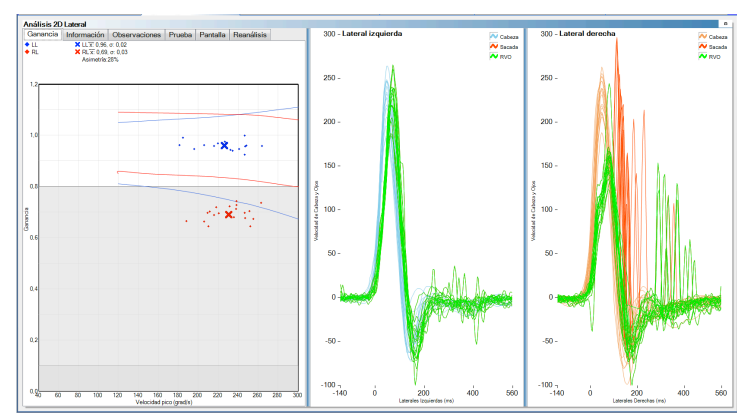

Figura 1. vHIT: análisis prequirúrgico donde se muestran ganancias de 0,6 en el lado enfermo.

Tras revisiones de su patología de base por parte del servicio de medicina interna, neurología y endocrinología, con control adecuado y sin presentar complicaciones derivadas de dichas patologías, se evaluaron y expusieron los riesgos y beneficios al paciente y se decidió 
actuar sobre el oído derecho optando por realizar una laberintectomía en el oído derecho y la colocación de un IC simultáneamente en el mismo oído, ante la previsión de una caída mayor en la audición del oído izquierdo. El paciente firmó el consentimiento informado de dicho procedimiento quirúrgico.

Técnicamente se realizó una laberintectomía clásica, exponiendo las ampollas de los tres canales y utrículo, con extirpación del epitelio sensorial y colocación de gentamicina en el lecho. El implante colocado en el oído derecho fue el modelo HiRes 90k Advantage HiFocus MS Electrode de Advanced Bionics. La inserción fue completa y la telemetría mostró respuesta en todos los electrodos (Figura 2).

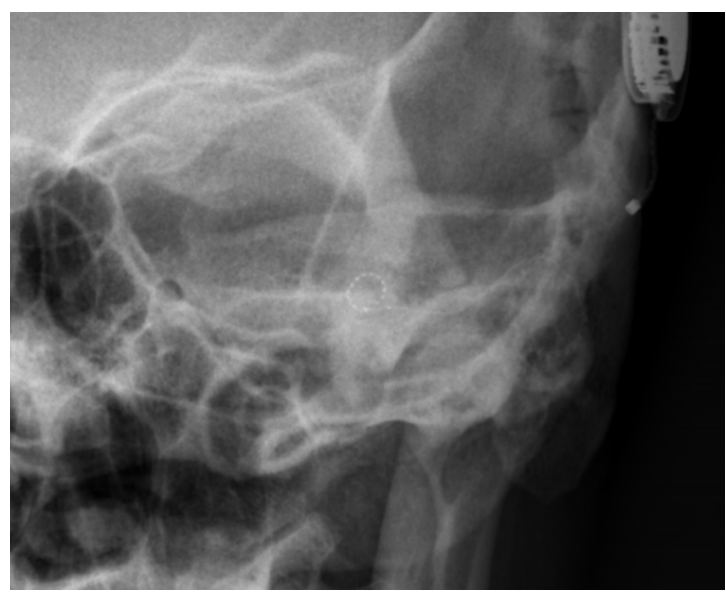

Figura 2. Radiografía simple en proyección de Stenvers que muestra implantación completa del IC.

El paciente dejó de presentar crisis de Tumarkin y la activación del IC fue satisfactoria; presentando una ganancia de 0,3 en el vHIT (Figura 3) y una audición con media de las frecuencias centrales de $40 \mathrm{~dB}$ y una inteligibilidad del $90 \%$ a $70 \mathrm{~dB}$ tras dos meses de activación. Tres meses tras la realización de la LQx y el IC, el paciente comenzó a presentar crisis de Tumarkin del oído izquierdo y algunas crisis vertiginosas con aumento de su pérdida auditiva hasta una hipoacusia severa. En el momento de enviar este artículo, está recibiendo tratamiento con gentamicina intratimpánica en oído izquierdo.

\section{DISCUSIÓN}

La base del tratamiento para la EM tiene como objetivo controlar las crisis con preservación de la función, pero en muchos pacientes los ataques de vértigo sólo cesan cuando toda la función coclear y vestibular ha sido destruida por el proceso de la enfermedad o los tratamientos empleados [25]. A esto se suma la incapacidad para predecir la historia natural de la enfermedad en cada paciente, pues una estrategia agresiva corre el riesgo de sobretratar al paciente que hubiera podido resolverse sin tal intervención, pero esa resolución natural podría resultar en años de padecimiento de incapacitantes y repetidas crisis vertiginosas con el resultado final de pérdida de función bilateral vestibular y auditiva. El clínico y el paciente deben evaluar el riesgo potencial y los beneficios de las distintas opciones [18].

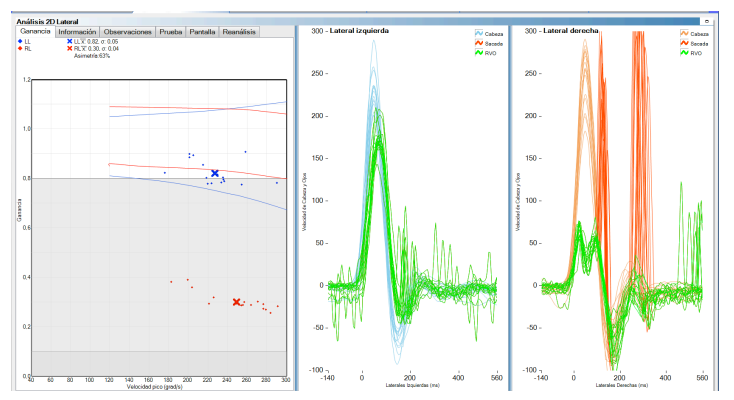

Figura 3. vHIT: análisis postquirúrgico donde se muestran ganancias de 0,3 en el lado enfermo.

En varios pacientes, las medidas conservadoras fallan y se han de considerar opciones más destructivas. No cabe duda que la LQx es un efectivo método de control y prevención de los ataques recurrentes de vértigo causados por EM. Los resultados confirman que resuelve las crisis en casi todos los pacientes con EM intratable [24]. Produce una sordera profunda y está típicamente reservada para pacientes que tienen poca o ninguna audición en el lado afecto y un lado contralateral útil [25].

La dificultad surge cuando los pacientes desarrollan la EM del segundo oído. Tradicionalmente, el tratamiento destructivo para un oído único por lo general estaría contraindicado. La excepción a esto es una sección del nervio vestibular que se utiliza con poca frecuencia debido a los riesgos significativos que tiene un procedimiento intracraneal además del riesgo de daño del nervio coclear. Lo que sí sabemos es que los pacientes que desarrollan una enfermedad de Ménière bilateral y en el proceso pierden audición útil residual pueden ser tratados con éxito con el implante coclear [25]. 
EI IC es la terapia indicada para restaurar la percepción auditiva en el oído sordo por EM. En un estudio realizado por McRackan et al. en 2014 se muestra que los pacientes que habían sido sometidos previamente a la cirugía de ablación por sus síntomas del Ménière tienen mejores resultados auditivos que los que no se han sometido a la cirugía no ablativa. Además, los pacientes con EM activa en el momento de colocación del IC es probable que tengan postoperatorio mejoría sintomática en sus síntomas vestibulares [14].

Aunque factores como la edad de implantación, la duración de la sordera y el proceso se hayan identificado como influyentes en IC [26], para su éxito en la EM, el grado de daño en el nervio coclear por la EM debe ser mínimo para permitir la conducción desde el IC al núcleo olivar superior [14]

Numerosos estudios indican que los restos cocleares son responsables de la estimulación eléctrica tras la LQx y analizan las modificaciones histopatológicas de los huesos temporales en pacientes con EM mostrando que la degeneración neuronal y de las células del ganglio espiral requeridas es rara, con lo que se esperará encontrar buenos resultados para el IC en EM tras la LQx. La severidad de la disminución de audición no se correlaciona siempre con las neuronas supervivientes en pacientes con EM [27]. Otros estudios demuestran que las modificaciones histológicas por fibrosis que se pueden producir en la cóclea tras la LQx pueden dificultar en un futuro la colocación de la guía de electrodos [19, 25]. Por ello y para evitar una segunda intervención, se decidió en este paciente realizar ambas en el mismo acto quirúrgico.

Dado el estado avanzado de la EM, las fluctuaciones, a menudo, se pueden resolver mediante la reprogramación subsiguiente [28]. La fluctuación tras IC, claramente demostrada en EM, es concomitante con episodios recurrentes de enfermedad sugiriendo el papel del hídrops en el deterioro de la capacidad auditiva que puede alterar la posición del electrodo intracoclear o que el escenario no sea adecuado por cicatrices, fibrosis y osificación, o bien, porque afecte directamente a las aferencias o a las propias neuronas del ganglio espiral [27, 28].

Una ventaja de tener un IC funcionante en el oído sordo inicial permitirá al paciente continuar oyendo aunque el contralateral se deteriore [24].
La cuestión es plantear cuándo efectuar las dos intervenciones, si conjuntamente o separadas en el tiempo y realizar la segunda según el curso de la enfermedad. En el pasado, se proponía que el IC tras LQx tendría un limitado éxito pues se asumía la discapacidad de los elementos neurales auditivos, pero posteriores estudios han evidenciado que la LQx no necesariamente conlleva a una destrucción de la vía auditiva [19, 29] con lo cual no es una contraindicación para el IC [28].

Así, IC con LQx simultánea no sólo alivia los ataques vertiginosos, también la audición. Como hemos visto, y dependiendo de los resultados a largo plazo de la audición tras IC, puede ser razonable ampliar la indicación de LQx a pacientes con vértigo intratable y audición residual [30, 31].

Esto es lo que nos planteamos en el caso presentado, tras frecuentes crisis de vértigo y de Tumarkin no controlables con fármacos o terapias intratimpánicas y una pérdida de audición unilateral con fluctuaciones contralaterales, se decidió realizar la laberintectomía con la colocación de IC en el oído causante de sus crisis de forma simultánea, con la previsión de que el lado contralateral se vea afectado en el futuro, ejemplo del planteamiento del IC en EM bilateral.

EI IC en pacientes que han sido sometidos previamente o simultáneamente a una LQx no es un concepto nuevo. El Dr. Brackmann fue el primero en realizar un IC en un paciente previamente laberintectomizado y demostrar excitabilidad eléctrica en dicho oído en 1975 [32, 33]. Zwolan fue el primero en realizar la LQx y colocación del IC en el mismo acto quirúrgico [29] y Lustig mostró en un estudio los resultados de nueve pacientes con IC para resolver hipoacusia profunda tras una larga evolución de la EM bilateral [25].

Las ventajas de realizar laberintectomía con IC simultáneo en pacientes con hipoacusia neurosensorial profunda o severa y crisis de vértigo intratables son:

- Reducir riesgos asociados con otro proceso quirúrgico y anestésico [24].

- La laberintectomía quirúrgica puede conducir a cicatrices en los tejidos blandos o incluso osificación de la cóclea la cual podría impedir la implantación más tarde [40], aunque esto no es una respuesta uniforme [19, 34]. 
- Finalmente, la implantación inmediata reduce el tiempo de sordera y la actuación con un IC está correlaciona con la duración de la sordera [35].

La única desventaja que podríamos encontrar se da en pacientes con audición residual prequirúrgica que no habrán experimentado las consecuencias de una sordera profunda y no apreciarán el beneficio del IC para la rehabilitación del nuevo déficit [24]. Además existen tres posibles razones que justifican el mareo crónico tras IC en EM [36]: que los síntomas sean reflejo de la enfermedad vestibular [37], que la implantación del electrodo cause daño en el órgano vestibular (fibrosis, hídrops con membrana sacular distorsionada, osteogénesis e incluso neuromas reactivos) $[38,39]$ o aquellas inherentes a la medidas de la función distintas para el sujeto y controles y para los clínicos.

\section{CONCLUSIONES}

Este caso es un ejemplo claro del enfoque terapéutico en un paciente con una previsión de HNS bilateral secundaria a EM bilateral. La combinación de LQx e IC es un excelente tratamiento para pacientes con EM y sordera unilateral. De forma simultánea es un método eficaz para el tratamiento del vértigo y rehabilitación del sistema auditivo requiriéndose un período de recuperación posterior para permitir la compensación vestibular, que a su vez, se ve influenciada por la edad y actividad física de los pacientes.

\section{BIBLIOGRAFÍA}

1. Anderson JP, Harris JP. Impact of Ménière's disease on quality of life. Otol Neurotol. 2001;22:888-94.

2. Stahle J, Stahle C, Anerberg IK. Incidence of Ménière's disease. Arch Otolaryngol. 1978;104:99-102.

3. Nakee K, Komatuzaki K. Epidemiological study of Ménière's disease. Pract Otol (Kyoto). 1984;69:1783-8.

4. Tokumaau K, Tashiro N, Goto K, et al. Incidence and prevalence of Ménière's disease in Aagamihara City, Kanagawaken. Pract Otol (Kyoto). 1983;1:1165-75.
5. Kotimaki J, Sorri M, Aantaa E, Nuutinen $\mathrm{J}$. Prevalence of Meniére's disease in Finland. Laryngoscope. 1999;109:74853.

6. Hallpike CS, Cairns H. Observations on the pathology of Ménière's syndrome: (Section of Otology). Proc R Soc Med. 1938;31:1317-36.

7. Committee on Hearing and Equilibrium. Committee on hearing and equilibrium guidelines for the diagnosis and evaluation of therapy in Ménière's disease. Otolaryngol Head Neck Surg. 1995;113:181-5.

8. Stahle J. Advanced Ménière's disease.A study of 356 severely disabled patients. Acta Otolaryngol. 1976;31:113-9.

9. Shojaku H, Watanabe $\mathrm{Y}$, Mizukoshi K, et al. Epidemiological study of severe cases of Ménière's disease in Japan. Acta Otolaryngol Suppl. 1995;520:4158.

10. Paparella MM, Griebie MS. Bilaterality of Ménière's disease. Acta Otolaryngol (Stockh). 1984;97:233-7.

11. Iseli C, Gibson W. A comparison of three methods of using transtympanic electrocochleography for diagnosis of Ménière's disease. Acta Otolaryngol. 2009;26:1-8.

12. Lin MY, Timmer FC, Oriel BS et al. Vestibular evoked myogenic potencials (VEMP) can detect asymptomtic saccular hydrops. Laryngoscope. 2006;116:987-92.

13. Fukuoka $\mathrm{H}$, Tsukada $\mathrm{K}$, Miyagawa $\mathrm{M}$, et al. Semi- quantitative evaluation of endolymphatic hydrops by bilateral intratympanic gadolinium-based contrast agent administration with MRI for Ménière's disease. Acta Otolaryngol. 2009;10:1-7.

14. McRackan T.R, Gifford R.N, Kahue C.H, Dwyer R, Labadie R.F, Wanna G.B, Haynes D.S, Bennett M.L. Cochlear Implantation in Ménière's Disease Patients. Otol Neurotol. 2014;35:421-5. 
15. Mancini F, Catalani M, Carru M, Monti B. History of Ménière's disease and its clinical presentation. Otolaryngol Clin North Am. 2002;35:565-80.

16. Arndt S, Aschendorff A, Laszig R, et al. Comparison of pseudobinaural hearing to real binaural hearing rehabilitation after cochlear implantation in patients with unilateral deafness and tinnitus. Otol Neurotol. 2011;32:39-47.

17. Amoodi HA, Mick PT, Shipp DB, et al. The effects of unilateral cochlear implantation on the tinnitus handicap inventory and the influence on quality of life. Laryngocope. 2011;121:1536-40.

18. Samuel A. C. MacKeith, MBChB, FRCS, Ian D. Bottrill, MBBS, FRCS, James D. Ramsden, FRCS. Simultaneous Labyrinthectomy With Cochlear Implantation in Patients With Bilateral Ménière's Disease. Ann Otol Rhinol Laryngol. 2014;123:485-9.

19. Douglas A Chen, Fred H. Linthicum JR, Frankin M Ritzer. Cochlear histopathology in the labyrinthectomized ear: implications for cochlear implantation. Laryngoscope. 1988;98:1170-2.

20. Pérez Fernández $\mathrm{N}$, Montes Jovellar L, Cervera Paz J et al. Auditory and vestibular assessment of patients with Meniere's disease who suffer Tumarkin attacks. Audiol Neurotol. 2010;15:399406.

21. Kentala E, Havia M, Pykko I, Short-lasting drop attacks in Ménière's disease. Otolaryngol Head Neck Surg. 2001;124:526-30.

22. Nguyen KD, Minor LB, Delia Santina CC et al. Vestibular function and vertigo control after intratympanic gentamicin for Ménière's disease. Audiol Neurotol. 2009:14:361-72.

23. Teufert KB, Doherty J. Endolymphatic sac shunt, labyrinthectomy and vestibular nerve section in Ménière's disease. Otolaryngol Clin North Am. 2010;43:1091-111.
24. Marlan R, Hansen, Bruce J. Gantz and Camille Dunn. Outcomes following cochlear implantation for patients with single-sided deafness, including those with recalcitrant Ménière's disease. Otol Neurotol. 2013;34:1681-7.

25. Lustig LR, Yeagle J, Niparko JK, Minor LB. Cochlear implantation in patients with bilateral Ménière's syndrome. Otol Neurotol. 2003;24:397-403.

26. Waltzman SB, Cosetti MK. Outcomes in cochlear implantation: variables affecting performance in adults and children. Otolaryngol Clin North Am. 2012;45:155-71.

27. Nadol J. Histologic considerations in implant patients. Arch Otolayngol Head Neck Surg. 1984;1:60-3.

28. Ravi N. Samy, Lisa Houston, Michael Scott, Daniel I. Cochlear implantation in patients with Ménière's Disease. Cochlear Implants Int. 2015;16:208-12.

29. Zwolan T.A, Sheoard N.T and Niparko J.K. Labyrinthectomy with cochlear implantation. Am J Otol. 1993;14:220-3.

30. Clemmens C, Ruckenstein M. Characteristics of patientswith unilateral and bilateral Ménière's disease. Otol Neurotol. 2012; 33:1266-9.

31. Belinchon A, Peres-GarriguesH,Tenias JM. Evolution of symptoms in Ménière's disease. Audiol Neurotol. 2012;17:12632.

32. Kveton JF, Abbott C, April M, Drumheller G, Cohen N, Poe DS. Cochlear implantation after transmastoid labyrinthectomy. Laryngoscope. 1989;99:610-613.

33. Eddington DK, Dobelle WG, Brackmann DE, Mladejovsky MG, Parkin JL. Auditory prostheses research with multiple cannel intracochlear stimulation in man. Ann Otol Rhinol Laryngol [Suppl]. 1978;53:1-39.

34. Osborn HA. Yeung R, Lin VY. Delayed cochlear implantation after surgical labyrinthectomy. J Laryngol Otol. 2012;126:63-5. 
35. Rubinstein JT, Parkinson WS, Tyler RS, et al. Residual speech recognition and cochlear implant performance: effects of implantation criteria. Am J Otol. 1999;20: 445-52.

36. Mick P, Amoodi H, Arnoldner C. Cochlear implantation in patients with advanced Ménière's disease. Otol Neurotol. 2014;35:1172-8.

37. Green JD, Blum DJ, Harner SG. Longitudinal followup of patients with Ménière's disease. Otolaryngol Head Neck Surg 1991;104:7838.
38. Batuecas-Caletrío A, Klumpp M, Santa Cruz-Ruiz S, Benito-González F, González-Sánchez E, Arriaga M. Vestibular function in cochlear implantation: Correlating objectiveness and subjectiveness. Laryngoscope 2015;125:2371-5.

39. Tien HC, Linthicum FH. Histopathologic changes in the vestibule after cochlear implantation. Otolaryngol Head Neck Surg. 2002;127:260-4. 